\title{
Dosage simultané de huit glycols dans les milieux biologiques par chromatographie en phase gazeuse couplée à la spectrométrie de masse
}

\section{Determination of eight glycols in biological specimens by gas chromatography-mass spectrometry}

\section{Jean-Pierre GOULLÉ*, Vincent GEMBUS, Christian LACROIX}

Laboratoire de Pharmacocinétique et de Toxicologie Cliniques, Groupe Hospitalier - BP 24 76083 LE HAVRE CEDEX - Tél : 0232733218 - Fax : 0232733238

* Auteur à qui adresser la correspondance : Docteur Jean-Pierre GOULLÉ, Laboratoire de Pharmacocinétique et de Toxicologie Cliniques, Groupe Hospitalier Jacques Monod - BP 24 - 76083 LE HAVRE Tél : 0232733223 - Fax : 0232733238 - E-mail : jgoulle@ch-havre.fr

\section{RÉSUMÉ}

L'objectif de ce travail est de proposer une technique de dosage des glycols dans les milieux biologiques, par chromatographie en phase gazeuse couplée à la spectrométrie de masse : éthylène glycol $=E G ;$ diéthylène glycol $=D E G$; 1,3-propanediol $=1,3-P D ; 1,2$-propanediol $=1,2-P D ; 1,2-$ butanediol $=1,2-B D ; 2,3$-butanediol $=2,3-B D ;$ triéthylène glycol $=T E G ;$ hexylène glycol $=H X G$. Le dosage est effectué par chromatographie en phase gazeuse couplée à la spectrométrie de masse à l'aide d'un injecteur de type splitsplitless et d'une colonne classique de type DBSMS. La prise d'essai est constituée par $20 \mu l$ d'échantillon biologique préalablement dilué au dixième. Après déprotéinisation de l'échantillon par l'acétonitrile, les glycols sont silylés en mono ou di dérivés TMS. La séparation chromatographique des dérivés obtenus est suffisante, elle permet la quantifica-

\section{SUMMARY}

A simple extraction and derivatization procedure for the analysis of eight glycols (ethylene gycol $=E G$; diethylene glycol $=D E G ; 1,3$-propanediol $=1,3-P D ; 1,2$-propanediol $=1,2-P D ; 1,2$-butanediol $=1,2-B D ; 2,3$-butanediol $=$ $2,3-B D$; triethylene glycol $=T E G$; hexylene glycol $=H X G$ ) using a $20 \mu \mathrm{l}$ ten fold dilution serum or blood sample is described. Following deproteinisation with acetonitrile, derivatization to its mono, di or tri TMS derivative, the glycols were detected using gas chromatography-electron impact mass spectrometry equipped with a split-splitless inlet and a $D B-5 M S$ column in the scan mode from 40 to 500 uma. Gamma-hydroxybuturate $d_{6}\left(G H B-d_{6}\right)$ was used as the internal standard. With one quantitation ion and two confirmation ions, the limit of detection and the limit of quantitation serum ranged respectively from $0,7 \mathrm{mg} / \mathrm{l}$ for $E G$ to $8,5 \mathrm{mg} / \mathrm{l}$ 
tion simultanée des 8 glycols. Les acquisitions sont réalisées en mode courant ionique total de 40 à 500 uma. Le gammahydroxybutyrate $d_{6}\left(G H B-d_{6}\right)$ est utilisé comme étalon interne. Après extraction d'un ion de quantification et de deux ions de confirmation, la limite de détection et la limite de quantification s'échelonnent respectivement de $0,7 \mathrm{mg} / \mathrm{l}$ pour l'EG à $8,5 \mathrm{mg} / \mathrm{l}$ pour le TEG et de $1,3 \mathrm{mg} / \mathrm{l}$ pour l'EG à 18,2 $m g / l$ pour le 1,2-PD. Dans le sérum et le sang, une réponse linéaire est obtenue entre 1 à 18 selon le glycol et $800 \mathrm{mg} / \mathrm{l}$. Pour une concentration de $400 \mathrm{mg} / \mathrm{l}$, la répétabilité et la reproductibilité ( $n=8$ et 10$)$ varient respectivement de 1,9 $\%$ pour le TEG à 4,9\% pour le 1,2-PD (11,8\% pour l'HXG), et de 3,5\% pour le DEG à $9,0 \%$ pour le 2,3-BD (20,4\% pour l'HXG). La méthode a été appliquée avec succès au plasma et au sang total. Cette technique complète utilement la méthode de dosage enzymatique de l'EG. Elle permet, outre la confirmation spécifique de la présence d'EG, de quantifier simultanément sept autres glycols.

\section{MOTS-CLÉS}

Glycols, échantillons biologiques, chromatographie gazeuse, spectrométrie de masse.

\section{Introduction}

L'intoxication par les glycols est un sujet de préoccupation majeure en toxicologie clinique en raison de la gravité potentielle de ces intoxications. Les intoxications par l'éthylène glycol sont de loin les plus fréquentes. En l'absence de traitement antidotique spécifique, le pronostic vital peut être engagé avec l'absorption de quelques dizaines de millilitres de liquide. Le dosage de l'éthylène glycol en urgence est de ce fait extrêmement utile pour valider une suspicion d'intoxication par ce produit dans un contexte clinique particulier avec des perturbations biologiques évocatrices : acidose sans corps cétoniques ni lactates, présence d'un «trou anionique». La gravité de l'intoxication n'est pas due à l'éthylène glycol lui même mais aux métabolites toxiques qui se forment sous l'effet de l'alcool déshydrogénase hépatique : acide glycolique, acide oxalique. Le traitement antidotique spécifique utilisant le 4-méthylpyrazole (Fomépizole $®)$ bloque cette transformation métabolique toxique $(1,2)$. La réponse analytique en urgence est classiquement effectuée par le dosage enzymatique de l'éthylène glycol (3-6) sur automate. Malheureusement cette technique bien adaptée pour le dosage sérique ou plasmatique ne peut être appliquée directement au sang total, une défécation est nécessaire. Par ailleurs, les critères d'identification et de quantification de la méthode ne répondent pas aux exigences actuelles de la médecine légale. Enfin, d'autres glycols peuvent être à l'origine d'intoxications graves et ne peuvent être détectés par voie enzymatique. Parmi eux le diéthylène glycol peut s'avérer très toxique, pouvant être à l'origine d'une insuffisance for TEG and from 1,3 $\mathrm{mg} / \mathrm{l}$ for $E G$ to $18,2 \mathrm{mg} / \mathrm{l}$ for 1,2-PD. A linear response was observed over the concentration range from $1-18$ to $800 \mathrm{mg} / \mathrm{l}$ for serum and blood. Coefficients of variation for both intra-assay precision and interassay reproductibility ranged respectively between $1,9 \%$ for TEG to $4,9 \%$ for $1,2-P D(11,8 \%$ for $H X G)$ and $3,5 \%$ for $D E G$ to $9,0 \%$ for 2,3-BD (20,4\% for $H X G)$ at the $400 \mathrm{mg} / \mathrm{l}$ serum level. The method was applied to plasma and whole blood.

\section{KEY-WORDS}

Glycols, gas chromatography, mass spectrometry.

rénale sévère comme cela a été montré au Bengladesh (7) où 429 enfants ont été hospitalisés sur une période de 3 ans, $21 \%$ d'entre eux présentant une insuffisance rénale en raison de la présence de diéthylène glycol dans un élixir à base de paracétamol. D'autres glycols sont couramment utilisés dans l'industrie chimique, dans des préparations commerciales diverses, ou entrent dans la composition de liquides de frein et peuvent être responsables d'intoxications professionnelles ou accidentelles voire utilisés lors de tentatives de suicide (8). Ce sont les raisons pour lesquelles il nous est apparu nécessaire de développer une technique de dosage à la fois relativement rapide et spécifique faisant appel à la chromatographie en phase gazeuse couplée à la spectrométrie de masse (CG/SM).

De nombreuses techniques de dosage des glycols ont été décrites depuis 20 ans par chromatographie en phase gazeuse. Parmi elles, celle de Porter (9) faisant appel à une dérivation par le phénylboronate est encore largement utilisée en raison de ses qualités, malheureusement, elle ne permet de doser que l'éthylène glycol après détection par ionisation de flammes. De très nombreuses autres méthodes ont été publiées permettant de quantifier d'autres glycols, le diéthylène glycol le plus souvent, voire simultanément des alcools ; mais elles font toujours appel à des colonnes ou à des injecteurs spécifiques du fait des caractéristiques physico-chimiques particulières (10-14). Peu de méthodes utilisent la spectrométrie de masse après dérivation $(15,16)$. La technique proposée par Maurer (16) est très intéressante en raison de sa haute spécificité mais elle est relativement complexe et ne permet de doser que l'éthylène glycol et de le diéthylène glycol. 


\section{Matériel et méthode Réactifs}

Le méthanol, l'acétonitrile sont de qualité chromatographie liquide haute performance (Carlo Erba, Val de Reuil, France). L'EG et le 2,3-butanediol (2,3-B) proviennent respectivement de chez Aldrich (Milwaukee, USA) et de chez Sigma (St Louis, USA). Tous les autres glycols ont été acquis chez Sigma-Aldrich (Steinheim, Allemagne). Le gamma-hydroxybutyrate d6 (GHB-d6) et le N,O-bis(triméthylsilyl)trifluoroacétamide contenant $1 \%$ de triméthylchlorosilane (BSTFA/TMCS) ont pour origine respective Radian (Austin, USA) et Interchim (Monluçon, France).

\section{Étalons et standards}

L'étalon interne servant à effectuer le dosage des glycols (GHB-d6) est préparé dans le méthanol à partir d'une dilution au 1/10è d'une solution mère à $100 \mathrm{mg} / \mathrm{l}$ pour obtenir une concentration finale de $10 \mathrm{mg} / \mathrm{l}$. Une solution de travail contenant le mélange des glycols à une concentration de $10 \mathrm{~g} / \mathrm{l}$ est préparée par pesée de $100 \mathrm{mg}$ de chacun des constituants du mélange dans une fiole jaugée de $10 \mathrm{ml}$ que l'on complète avec de l'eau distillée. Des solutions filles sont préparées en diluant 100,400 et $800 \mu \mathrm{l}$ de la solution de travail avec $1 \mathrm{ml}$ d'eau distillée. Ces solutions sont diluées au 1/10è dans un sérum ou dans du sang exempt de glycol. Les solutions étalons obtenues ont les concentrations suivantes: $100,400,800 \mathrm{mg} / \mathrm{l}$.

\section{Méthode d'extraction}

Dans un tube Eppendorf on ajoute à $20 \mu \mathrm{l}$ d'une solution au $1 / 10$ è de l'échantillon à analyser $50 \mu \mathrm{l}$ d'étalon interne deutéré (GHB-d6) et $45 \mu$ l d'acétonitrile. Le mélange est agité au vortex pendant 10 secondes. Chaque tube est centrifugé à 10900 tours pendant 4 minutes. La fraction liquide est transférée dans un flacon propre de dérivation puis évaporé sous azote à température ambiante. Les extraits sont dérivés avec $50 \mu \mathrm{l}$ de BSTFA/TMCS pendant 20 minutes à $70^{\circ} \mathrm{C}$. Après refroidissement les échantillons sont placés dans des flacons pour le préleveur automatique du chromatographe en phase gazeuse.

\section{Appareillage}

Une fraction de $1 \mu \mathrm{l}$ de l'échantillon dérivé est injectée dans un chromatographe en phase gazeuse HewlettPackard (modèle HP6890) relié à un détecteur de masse (modèle HP5973). L'appareil fonctionne en mode impact électronique et les acquisitions sont effectuées sur le chromatographe en phase gazeuse couplé au srectromètre de masse (CG/SM) de 40 à 500 uma. L'ensemble des données est enregistré sur une station informatique. Le chromatographe est équipé d'une colonne capillaire de type DB-5MS $5 \%$ de phényl et $95 \%$ de méthylsiloxane $(30 \mathrm{~m} \times 0,25 \mathrm{~mm}$ de diamètre interne et $0,25 \mu \mathrm{m}$ d'épaisseur de film). Le gaz vecteur est de l'hélium, son débit constant est de $1 \mathrm{ml}$ par minute. Les injections sont réalisées en mode splitless pulsé et la température de l'injecteur est de $230^{\circ} \mathrm{C}$. La température initiale du four est de $80^{\circ} \mathrm{C}$ pendant 1 minute puis montée à $140^{\circ} \mathrm{C}$ à la vitesse de $5^{\circ} \mathrm{C}$ par minute et enfin à $240^{\circ} \mathrm{C}$ à la vitesse de $40^{\circ} \mathrm{C}$ par minute. Cette température est maintenue pendant 5 minutes. La durée totale d'acquisition est de 20,5 minutes.

\section{Validation de la méthode}

La détermination des glycols dans le sérum est effectuée à l'aide d'un étalonnage interne avec le GHB-d6. Une réponse linéaire est obtenue pour le dosage de 8 glycols ajoutés simultanément dans un sérum aux concentrations suivantes : $0,125,250,500,750 \mathrm{mg} / \mathrm{l}$. La limite de détection (LD) est définie comme la valeur moyenne du blanc à laquelle on ajoute trois écarts types (ET), à partir du bruit de fond mesuré sur 30 échantillons ne contenant pas de glycol. La limite de quantification (LQ) est définie comme la valeur moyenne du bruit de fond à laquelle on ajoute 10 ET. L'échantillon de sérum du contrôle de qualité, organisé par la Société Française de Toxicologie Analytique (SFTA, Dr DEVEAUX), a été analysé par cette technique.

\section{Résultats}

Le tableau I montre le poids moléculaire des différents dérivés obtenus et les ions caractéristiques et le temps de rétention pour chaque glycol ainsi que pour l'étalon interne deutéré. Les courbes d'étalonnage permettent d'établir la relation entre la réponse obtenue et la concentration pour chacun des glycols. Chaque courbe d'étalonnage est reportée figure 1 . Le coefficient de corrélation est supérieur à 0,99 pour chaque glycol. La LD et la LQ pour une prise d'essai de $20 \mu \mathrm{l}(2 \mu \mathrm{l}$ d'échantillon dilué au 1/10è) s'échelonnent respectivement de $0,7 \mathrm{mg} / \mathrm{l}$ pour l'EG à $8,5 \mathrm{mg} / \mathrm{l}$ pour le triéthylène glycol (TEG) et de $1,3 \mathrm{mg} / \mathrm{l}$ pour l'EG à $18,2 \mathrm{mg} / \mathrm{l}$ pour le 1,2-propanediol (1,2-PD). Les autres résultats sont reportés tableau II. La répétabilité et la reproductibilité ont été déterminés aux 3 concentrations suivantes pour chaque glycol : 100, 400 et $800 \mathrm{mg} / \mathrm{l}$. Les résultats sont regroupés tableau III. La linéarité de la technique a été validée pour des concentrations en glycols de $1000 \mathrm{mg} / \mathrm{l}$. Le résultat obtenu pour le contrôle de qualité de la SFTA est reporté tableau IV. 
Tableau I : Masse des dérivés (M), ions dominants et temps de rétention pour les 8 glycols et l'étalon interne.

\begin{tabular}{|l|c|c|c|}
\hline Dérivé & {$[\mathbf{M}]$} & Ions $(\mathbf{m} / \mathbf{z})$ & $\begin{array}{c}\text { Temps } \\
\text { Rétention } \\
(\mathbf{m i n})\end{array}$ \\
\hline di-TMS EG & $\mathbf{2 0 6}$ & $\mathbf{1 9 1 , 1 3 3 , 1 0 3}$ & $\mathbf{4 , 6}$ \\
di-TMS DEG & $\mathbf{2 5 0}$ & $\mathbf{2 3 5}, 189,133$ & $\mathbf{9 , 5}$ \\
di-TMS TEG & $\mathbf{2 9 4}$ & $\mathbf{1 6 1 , 2 7 9 , 2 7 5}$ & $\mathbf{1 4 , 4 5}$ \\
di-TMS 1,2-PD & $\mathbf{2 2 0}$ & $\mathbf{2 0 5}, 175,133$ & $\mathbf{4 , 6}$ \\
di-TMS 1,3-PD & $\mathbf{2 2 0}$ & $\mathbf{2 0 5}, 189,174,130$ & $\mathbf{5 , 5}$ \\
di-TMS 1,2-BD & $\mathbf{2 3 4}$ & $\mathbf{2 1 9 , 1 7 7 , 1 3 1}$ & $\mathbf{6 , 3}$ \\
di-TMS 2,3-BD & $\mathbf{2 3 4}$ & $\mathbf{2 1 9}, 173,133$ & $\mathbf{5 , 3}$ \\
mono-TMS HXG & $\mathbf{1 9 0}$ & $\mathbf{1 1 9 , 1 7 5 , 1 5 7}$ & $\mathbf{5 , 2 8}$ \\
di-TMS GHB-D6* & $\mathbf{2 5 4}$ & $\mathbf{2 3 9 , 2 0 6 , 1 3 3}$ & $\mathbf{9 , 2 9}$ \\
\hline *étalon interne
\end{tabular}

Tableau II : Limite de détection (LD) et de quantification (LQ) des 8 glycols dans le sérum.

\begin{tabular}{|l|c|c|}
\hline Dérivé & LD $\mathbf{~ m g} / \mathbf{L}$ & LQ $\mathbf{~ m g} / \mathbf{L}$ \\
\hline di-TMS EG & 0,7 & 1,3 \\
di-TMS DEG & 7,9 & 16,6 \\
di-TMS TEG & 8,5 & 18,0 \\
di-TMS 1,2-PD & 8,0 & 18,2 \\
di-TMS 1,3-PD & 2,4 & 3,7 \\
di-TMS 1,2-BD & 6,9 & 14,4 \\
di-TMS 2,3-BD & 2,9 & 6,1 \\
mono-TMS HXG & 5,4 & 13,1 \\
\hline
\end{tabular}

Tableau III : Étude de la répétabilité et de la reproductibilité.

\section{Discussion}

Nous proposons une méthode qui consiste après une simple précipitation puis évaporation à dériver les glycols avec un agent de méthylation usuel. Elle présente des avantages déterminants sur les techniques décrites : prise d'essai réduite $(2 \mu \mathrm{l})$, emploi d'un injecteur classique de type split-splitless, d'une colonne de routine pour analyse toxicologique par CG/SM avec $5 \%$ de phénylméthylsilicone ; identification formelle des structures par spectrométrie de masse.

En ce qui concerne ces performances, les droites de régression permettant de relier les concentrations aux signaux obtenus sont parfaitement linéaires et le coefficient de corrélation est excellent puisqu'il est supérieur pour chaque glycol à 0,99 dans la gamme de concentration étudiée. Les LD et les LQ permettent non seulement de surveiller une intoxication aux glycols, mais également de mettre en évidence une faible exposition. Les coefficients de variation montrent que la répétabilité et la reproductibilité sont bonnes puisque pour 7 des 8 glycols, elles sont inférieures à $11 \%$. En ce concerne l'hexylène glycol (HXG), les coefficients de variation obtenus sont supérieurs puisqu'ils sont compris entre 10 et $20 \%$, mais comme il n'existe aucune autre méthode pour le quantifier, les résultats sont considérés comme corrects. La méthode décrite pour la détermination des 8 glycols est précise, sensible et reproductible. Elle peut être facilement appliquée aux différents liquides biologiques. Cette technique de

\begin{tabular}{|c|c|c|c|c|c|c|}
\hline \multirow[t]{2}{*}{ Dérivé } & \multicolumn{3}{|c|}{$\begin{array}{l}\text { Répétabilité } \\
\% \mathrm{CV}^{*}(\mathrm{n}=8)\end{array}$} & \multicolumn{3}{|c|}{$\begin{array}{c}\text { Reproductibilité } \\
\% \mathrm{CV}^{*}(\mathbf{n}=10)\end{array}$} \\
\hline & $100 \mathrm{mg} / \mathrm{L}$ & $400 \mathrm{mg} / \mathrm{L}$ & $800 \mathrm{mg} / \mathrm{L}$ & $100 \mathrm{mg} / \mathrm{L}$ & $400 \mathrm{mg} / \mathrm{L}$ & $800 \mathrm{mg} / \mathrm{L}$ \\
\hline di-TMS EG & 4,4 & 2,8 & 4,2 & 4,7 & 4,7 & 5,4 \\
\hline di-TMS DEG & 2,2 & 3,9 & 3,4 & 3,5 & 3,5 & 3,5 \\
\hline di-TMS TEG & 4,4 & 1,9 & 4,1 & 7,3 & 6,2 & 6,6 \\
\hline di-TMS 1,2-PD & 5,3 & 4,9 & 6,9 & 6,2 & 5,8 & 5,1 \\
\hline di-TMS 1,3-PD & 4,1 & 2,7 & 5,7 & 4,8 & 4,9 & 3,8 \\
\hline di-TMS 1,2-BD & 6,5 & 3,3 & 8,1 & 7,8 & 7,8 & 5,4 \\
\hline di-TMS 2,3-BD & 7,2 & 4,7 & 10,9 & 9,0 & 9,0 & 8,9 \\
\hline mono-TMS HXG & 10,8 & 11,8 & 20,1 & 20,6 & 20,4 & 18,7 \\
\hline
\end{tabular}

Tableau IV : Contrôle de qualité 2001 alcools et éthylène glycol organisé par la SFTA (Dr DEVEAUX).

\begin{tabular}{|l|c|}
\hline \multicolumn{1}{|c|}{ Technique utilisée } & Concentration en EG $(\mathrm{g} / \mathbf{l})$ \\
\hline Enzymologie $(\mathrm{n}=10)$ & 0,45 \\
Toutes techniques $(\mathrm{n}=35)$ & 0,40 \\
Technique proposée CG/SM & 0,37 \\
\hline
\end{tabular}

quantification ne remet pas en cause l'emploi de la méthode enzymatique qui permet de mesurer l'éthylène glycol très rapidement, avec une assez bonne précision, suffisante pour les besoins de la toxicologie d'urgence. En effet, cette technique peut être réalisée sur automate en 10 minutes ce qui n'est pas le cas de la méthode proposée, beaucoup plus longue. Cependant, la méthode chromatographique peut être considérée 

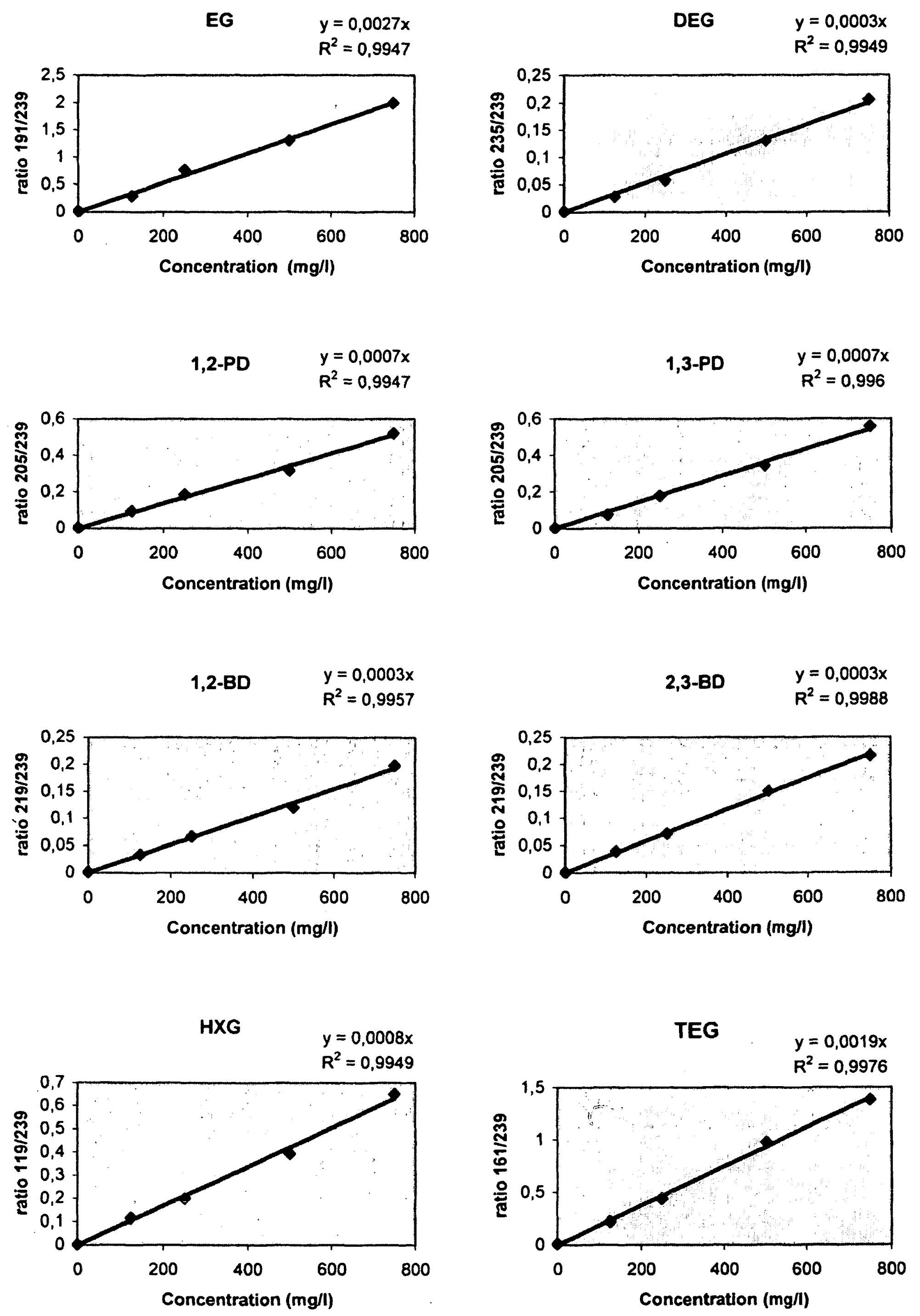

'igure 1 : Courbes d'étalonnage pour l'EG, le DEG, le 1,2-PD, le 1,3-PD, le 1,2-BD, le 2,3-BD, l'HXG et le TEG. 
comme une méthode complémentaire permettant en particulier d'identifier formellement la présence d'EG grâce au détecteur de masse. Cette technique permet par ailleurs de mettre en évidence et de quantifier d'autres glycols que l'EG, et d'obtenir les informations nécessaires pour mettre en œuvre un traitement approprié aux malades. De nombreuses autres méthodes ont été développées, en particulier par chromatographie en phase gazeuse couplée à une détection par ionisation de flamme (FID), mais celle-ci ne permet que la détermination de l'EG $(11,17)$. Pour quantifier d'autres glycols par chromatographie en phase gazeuse, des méthodes ont été décrites ; cependant, elles sont difficiles à mettre en œuvre compte-tenu de la nécessité d'utiliser des équipements spécifiques : colonnes spéciales, injecteurs particuliers $(9,12-14))$. En ce qui concerne les techniques par CG/SM, il est également indispensable d'utiliser des équipements spécifiques ou bien des dérivations particulières. L'ionisation chimique a également été décrite et la CG/SM fait également appel à des colonnes particulières ou des injecteurs spécifiques pour déterminer les différents glycols $(15,16)$. De plus la méthode proposée ne montre pas d'interférence entre les différents glycols qui peuvent être quantifiés simultanément et la gamme de mesure est adaptée à la détermination de concentrations toxiques ou pouvant avoir une conséquence mortelle en ce qui concerne les différents glycols.

\section{Conclusion}

La méthode décrite est facile à mettre en œuvre dans tous les laboratoires disposant d'un chromatographe en phase gazeuse couplé à un spectromètre de masse. Elle présente les avantages déterminants d'utiliser une technique de dérivation classique et de faire appel à la colonne de routine pour l'analyse toxicologique. Elle permet en outre de doser simultanément 8 glycols à partir d'une prise d'essai extrêmement réduite.

\section{Références}

1. Baud F.J., Bien D.V., Likforman J., Houzé P., Lambert C., Bismuth C. 4-methylpyrazole as an antidote in ethylene glycol and methanol poisoning. Geneva : World Healt Organisation. 1993.

2. Danel V., Hanna J. Actualité des traitements en toxicologie. $9^{\text {enne }}$ Congrès de la Société Française de Toxicologie Analytique. La Clusaz, 12-16 mars 2001.

3. Standefer J., Blackwell W. Enzymatic method for measuring ethylene glycol with a centrifugal analyzer. Clin Chem. 1991 ; 37 : 1734-1736.

4. Goullé J.P., Allion M.J., Anagnostides J. Ethylène glycol : intérêt d'une méthode enzymatique en toxicologie d'urgence. Toxicorama. $1995 ; 7: 16-20$.

5. Mahly M., Lardet G., Vallon J.J. Automated cobas mira kintetic enzymatic assay for ethylene glycol applied to emergency situations. J Anal Toxicol. 1994 ; 18 : 269-271.

6. Malandain H., Cano Y. Des méthodes enzymatiques simples, utilisables en garde, pour doser le méthanol, l'éthylène glycol et l'acide glycolique. Toxicorama. $1995 ; 7$ : 31-37.

7. Ballantyne B., Mans T., Syversen T. General and applied toxicology. Macmillam Reference LID. 1999; 3 : 1426-1427.

8. Nann N., Bajwa I., Tobin J., DasGupta A., SkeulaPerlman A., Callery R. A fatal case of ingestion of brake fluid containing diethylene and triethylene glycols. TIAFT bull. $1996 ; 26: 34-37$.

9. Porter W.H., Auansakul A. Gas-chromatographic determination of ethylene glyol in serum. Clin Chem. 1982 ; $28: 75-78$.

10. Gaillard Y., Gay-Montchamp J.P., Ollagnier M. Identification et dosages du méthanol, de l'éthanol et de l'éthylène glycol dans les liquides biologiques par chromatographie en phase gazeuse. Toxicorama. $1993 ; 4$ : 39-42.
11. Jonsson J.A., Eklund A., Molin L. Determination of ethylene glycol in postmortem blood by capillary gas chromatography. J Anal Toxicol. $1989 ; 13: 25-26$.

12. Livesey J.F., Perkins S.L., Tokessy N.E., Maddock M.J. Simultaneous determination of alcohols and ethylene glycol in serum by packed- or capillary-column gas chromatography. Clin Chem. 1995 ; 41 : 300-305.

13. Aarstad K., Dale O., Aakervik O., Ovrebo S., Zahlsen K. A rapid gas chromatographic method for the determination of ethylene glycol in serum and urine. J Anal Toxicol. $1993 ; 17: 218-221$.

14. Williams R., Shah S., Maggiore J., Erickson T. Simultaneous detection and quantitation of diethylene glycol, ethylene glycol and the toxic alcohols in serum using capillary column gas chromatogrpahy. J Anal Toxicol. $2000 ; 24: 621-626$.

15. Dasgupta A., Macaulay R. A novel derivatization of ethylene glycol from human serum using 4-carbethoxyhexafluorobutyryl chloride for unambigous gas chromatography-chemical ionization mass spectrometric identification and quantification. Am J Clin Pathol. 1995 ; 104 : 283-288.

16. Maurer H. Peters F., Paul L., Kraemer T. Validated gas chromatographic-mass spectrometric assay for determination of the antifreezes ethylene glycol and diethylene glycol in human plasma after microwave-assisted pivalylation. J Chromatogr B Biomed Sci Appl. 2001 ; 754 : 401-409.

17. LeGatt D., Tisdell R. Ethylene glycol quantification : avoid propylene glycol as internal standard. Clin Chem. $1990 ; 36: 1860-1861$. 